\title{
La Sarcoïdose Thyroïdienne :
}

\author{
H.El Ouahabi-M.Rchachi \\ Service d'Endocrinologie, Diabétologie Et Nutrition, CHU Hassan II Fès
}

\begin{abstract}
La sarcoüdose est une maladie inflammatoire granulomateuse. L'atteinte thyrö̈dienne est exceptionnellement isolée et rarement confirmée histologiquement. Il s'agit le plus souvent d'une atteinte purement morphologique: goitre simple ou nodule bénin. Les anomalies fonctionnelles sont plus rares: thyrö̈dites, hypothyrö̈die.

Nous rapportons le cas d'une patiente âgée de 52 ans qui présente une sarcoüdose thyrö̈dienne déclarée par un goitre euthyrö̈dien et confirmé à l'examen anatomopathologique.
\end{abstract}

Keywords: sarcoidose, thyrö̈de, granulome épithéloide

\section{Introduction}

La sarcoïdose est une maladie inflammatoire granulomateuse, avec une atteinte préférentielle des poumons et des ganglions lymphatiques médiastinaux. Parmi ses atteintes extra-thoraciques, l'atteinte des glandes endocrines et notamment de la thyroïde constitue une manifestation rare de la maladie, probablement sous-diagnostiquée, d'autant plus que l'infiltration thyroïdienne retentit peu sur la fonction de la glande. Nous rapportons une observation de localisation thyroïdienne de la sarcoïdose.

\section{Cas clinique:}

Patiente âgée de 52ans, suivi depuis 4 ans pour une sarcoïdose pulmonaire diagnostiquée sur des biopsies bronchiques étagées réalisées devant la survenue d'une dyspnée associée à des douleurs inter-scapulaires avec sur la TDM pulmonaire de multiples micronodules parenchymateux pulmonaires et des adénopathies latéro-trachéales et hilaires bilatérales. La patiente avait consulté dans notre formation pour une tuméfaction cervicale sans signes inflammatoires locaux ni altération de l'état général, chez qui l'exploration avait révélé un goitre multi-hétéronodulaire en euthyroïdie clinique et biologique.

Une thyroïdectomie totale a été indiquée chez la patiente .L'examen anatomopathologique de la pièce opératoire avait montré un infiltrat lymphoïde avec granulomes épithélioïdes et gigantocellulaires sans nécrose caséeuse en faveur d'une sarcoïdose thyrö̈dienne.

\section{Discussion}

Le terme sarcoïdose a été utilisé la première fois par un dermatologue Caesar Boeck en 1899 pour décrire des nodules cutanés caractérisés par des foyers compacts qui définissent le granulome épithéloide [1]. Il s’agit d'une maladie granulomateuse inflammatoire d'origine indéterminée.

Bien que la sarcoïdose puisse toucher plusieurs organes notamment les yeux et les reins, l'atteinte pulmonaire reste la plus courante. L'atteinte des glandes endocrines est extrêmement rare. Une atteinte multiglandulaire a été rapportée dans la littérature incluant une hypercalcémie, un hypopituitarisme et une sarcoïdose thyroïdienne [2].

La localisation thyroïdienne de la sarcoidose a été décrite la première fois en 1938 par Spencer et al [1]. Elle est exceptionnellement isolée : Des cas ont été signalés dans la littérature et rarement confirmées histologiquement [3]. Une association rare d'un cancer papillaire de la thyrö̈de avec des métastases ganglionnaires et une sarcoidose thyroïdienne a été rapportée [4]. Cliniquement, la fréquence de l'atteinte thyroidienne varie de $0,6 \%$ à $19 \%$ selon les études. Il s'agit le plus souvent d'une atteinte purement morphologique: goitre simple ou nodule bénin [5]. Les anomalies fonctionnelles sont plus rares: thyrö̈dites, hypothyroïdie. La positivité des anticorps quelle que soit la méthode utilisée est plus importante chez les patients atteints de sarcoïdose [6].

Les études autopsiques révèlent $1,3 \%$ à $4,2 \%$ de localisations thyroïdiennes chez l'adulte atteint de sarcoïdose sans notion d'atteinte clinique ou biologique [1]. L'intérêt du cas présent est l'association de la localisation thyroïdienne rare dans la sarcoïdose avec une preuve formelle par l'examen anatomopathologique du goitre. Le diagnostic formel de sarcoïdose thyroidienne est basé sur l'histologie. L'évolution se fait soit vers la guérison, soit vers l'apparition d'une fibrose avec dépôt de collagène et destruction du tissu sain. 


\section{Conclusion}

La sarcoïdose est une maladie systémique à localisations multiples. Son diagnostic est basé sur l'histologie. L'atteinte pulmonaire est la plus courante et précède généralement l'atteinte thyroidienne. Une sarcoïdose thyroidienne doit être évoquée devant toute atteinte thyroidienne associée à une atteinte pulmonaire.

\section{Références}

[1]. Spencer J, Warren S. Boeck's sarcoid: Report of a case with clinical diagnosis confirmed at autopsy. Arch Intern Med. 1938; 62:285-296

[2]. Bell NH. Endocrine complications of sarcoidosis. Endocrinol Metab Clin North Am 1991; 20:645-54.

[3]. Cabibi D, Di Vita G, La Spada E, Tripodo C, Patti R, Montalto G.. Thyroid sarcoidosis as a unique localization Thyroid. 2006;16:1175-1177.

[4]. Viswanath L, Pallade S, Krishnamurthy B, Naveen T, Preethi BL, Pramod KP, Reddy O, Padma G., Darier-Roussy sarcoidosis mimicking metastatic breast cancer, Case Rep. Oncol. 2 (2009) 251-254

[5]. Vailati A, Marena C, Aristia L, Sozzé E, Barosi G, Inglese V, Luisetti M, Bossolo PA.. Sarcoidosis of the thyroid: report of a case and a review of the literature. Sarcoidosis. 1993;10:66-68

[6]. Antonelli A, Fazzi P, Fallahi P, Ferrari SM, Grosso M, Boni G, Ferrannini E, Mariani G.. Thyroid uptake of 67Ga-citrate is associated with thyroid autoimmunity and hypothyroidism in patients with sarcoidosis. Eur J Nucl Med Mol Imaging. 2009;36:137143 